\title{
Energy-Efficient Resource Allocation for Industrial Cyber-Physical IoT Systems in 5G Era
}

\author{
Song Li, Member, IEEE, Qiang Ni, Senior Member, IEEE, Yanjing Sun, Member, IEEE, Geyong Min, \\ Member, IEEE, and Saba Al-Rubaye, Senior Member, IEEE
}

\begin{abstract}
Cyber-physical Internet of things system (CPIoTS), as an evolution of Internet of things (IoT), plays a significant role in industrial area to support the interoperability and interaction of various machines (e.g. sensors, actuators, and controllers) by providing seamless connectivity with low bandwidth requirement. The fifth generation (5G) is a key enabling technology to revolutionize the future of industrial CPIoTS. In this paper, a communication framework based on $5 \mathrm{G}$ is presented to support the deployment of CPIoTS with a central controller. Based on this framework, multiple sensors and actuators can establish communication links with the central controller in full-duplex mode. To accommodate the signal data in the available channel band, the resource allocation problem is formulated as a mixed integer non-convex programming problem, aiming to maximize the sum energy efficiency of CPIoTS. By introducing the transformation, we decompose the resource allocation problem into power allocation and channel allocation. Moreover, we consider an energy-efficient power allocation algorithm based on game theory and Dinkelbach's algorithm. Finally, to reduce the computational complexity, the channel allocation is modeled as a 3-dimensional matching problem, and solved by iterative Hungarian method with virtual devices (IHM-VD). A comparison is performed with well-known existing algorithms to demonstrate the performance of the proposed one. The simulation results validate the efficiency of our proposed model, which significantly outperforms other benchmark algorithms in terms of meeting the energy efficiency and the QoS requirements.
\end{abstract}

Index Terms-5G, cyber-physical IoT system, energy efficiency, full-duplex, resource allocation

\section{INTRODUCTION}

$\mathrm{M}$ ANY practical systems in the industrial area, such as smart grids, smart manufacturing and health-care systems, can be categorized as industrial cyber-physical Internet of things systems (CPIoTSs). CPIoTSs, which facilitate tight integration

Manuscript received August 23, 2017; revised December 29, 2017; accepted January 6,2018 . This work was supported in part by the National Natural Science Foundation of China under Grant 51504255,51504214 , in part by the EU FP7 CROWN Project under Grant PIRSES-GA-2013-610524.

$\mathrm{S}$. $\mathrm{Li}$ and $\mathrm{Y}$. Sun are with the School of Information and Control Engineering, China University of Mining and Technology, Xuzhou 221116, China (e-mail: lisong, yjsun@cumt.edu.cn).

Q. Ni is with the School of Computing and Communications, Lancaster University, Lancaster LA1 4WA, U.K (e-mail: q.ni@lancaster.ac.uk).

G. Min is with the Department of Computer Science, College of Engineering, Mathematics, and Physical Sciences, University of Exeter, Exeter, EX4 4QF, U.K. (e-mail: g.min@exeter.ac.uk).

S. Al-Rubaye is with the Quanta Technology, Toronto, M2M3T8, Canada (e-mail: saba.alrubaye@ieee.org). and coordination between various entities and physical processes through networks, can be considered as an evolution of Internet of things (IoT) [1].

The numbers of various IoT machines are expected to grow rapidly, as sensors and actuators are widely deployed in multiple applications, such as cyber security, automation, metering, health-care, utilities, and consumer electronics. According to [2], IoT will support the integration of approximately 5.5 million things every day by 2016, with estimation of 20.8 billion things by 2020 . The rapid increase of IoT machines brings some technical challenges in order to meet the complex requirements. For example, the existing wireless communication technologies with low-power are still under investigation to meet the energy-efficient requirement with high system reliability. Therefore, research efforts should be carried out to tackle the obstacle of CPIoTS to be deployed in practice, especially in industrial applications [3].

Among all possible wireless communication network solutions for CPIoTS, 5G is considered as a promising technology, which integrates entities, communications and control technologies [4]. Since 5G supports enhanced mobile broadband communications, ultra-reliable and low latency communications (URLLC) and massive machine-type communications (mMTC), it is clear that URLLC and mMTC in $5 \mathrm{G}$ are closely related to industrial CPIoTS. Thus $5 \mathrm{G}$ provides an ideal platform for communications in industrial CPIoTS. In such a 5G-enabled CPIoTS, one major technical challenge is how to design energy-efficient communication that can facilitate various resources while providing satisfactory QoS and supporting dynamic network environments.

To meet the critical QoS requirements in industrial CPIoTS, some advanced technologies are under investigation in $5 \mathrm{G}$, including device-to-device communication (D2D) and full-duplex communication [5]. D2D allows two adjacent mobile devices to establish a direct communication link in order to facilitate local mobile service [6][7]. In [6], the resource allocation for $\mathrm{D} 2 \mathrm{D}$ is investigated using a matching approach and an algorithm based on many-to-one matching with peer effects is proposed, which can achieve a two-sided exchange stable matching. On the other hand, full-duplex communications, as another promising technology in 5G, have the potential to provide significant spectral efficiency and energy efficiency gain, by allowing one node to receive and transmit signal using the same channel at the same time [8][9]. In a full-duplex cellular system, since the full-duplex base 
station suffers from self-interference and downlink users suffer from co-channel interference from uplink users, the resource allocation problem is considered as a major challenge. In [10], the sum-rate of a full-duplex multi-user system with one full-duplex base station and multiple half-duplex mobile users is maximized by joint user pairing, subchannel allocation, and power allocation. In [11], the full-duplex resource allocation problem is modeled as a non-cooperative game between uplink and downlink channels. An iterative algorithm is proposed to achieve Nash equilibrium. However, all papers mentioned above mainly focus on the spectral efficiency optimization.

Besides spectral efficiency, energy efficiency plays an important role in CPIoTS communications. Energy-efficient communication becomes an emerging technology, since most of wireless sensors and actuators in CPIoTS are battery powered and thus are fundamentally constrained by the energy due to the limited battery capacity and charging facilities [12]. Potential solutions for energy efficiency improvement include energy-efficient protocol design and energy-efficient resource allocation [13]-[15]. Focusing on the critical issue, resource allocation problem was investigated to maximizing energy efficiency in various wireless networks, including relay system [16], device-to-device communications [17]. However, the energy-efficiency issue for full-duplex communication in 5G-enabled CPIoTSs has not been investigated yet.

In this paper, we focus on energy-efficient resource allocation for the communication systems in 5G-enabled CPIoTSs. We consider a CPIoTS consisting of one full-duplex central controller, multiple sensors, and multiple actuators. The resource allocation problem is formulated as the maximization of sum energy efficiency of all sensors and actuators. The major contributions of this paper are summarized as follows:

(1) We propose a new communication framework based on $5 \mathrm{G}$ technology to support industrial CPIoTS, in which multiple sensors and actuators are connected with a full-duplex central controller. Specifically, the central control is allowed to operate in either half-duplex mode or full-duplex mode on each channel.

(2) We formulate the energy-efficient resource allocation problem in CPIoTS, considering the QoS requirement of each sensor and actuator in terms of minimum transmit rate. By introducing virtual devices into the CPIoTS, the resource allocation problem is converted into a generalized mixed integer nonlinear problem (MINLP).

(3) To tackle the resource allocation problem, we decompose the original optimization problem into power allocation and channel allocation to reduce the computational complexity. The energy-efficient power allocation of sensor and actuator allocated on each channel is solved based on game theoretical approach and Dinkelbach's algorithm considering different channel allocation cases. Then the channel allocation is modeled as a 3-dimensional matching among sensors, actuators and channels, and further solved by an iterative algorithm denoted as IHM-VD.

The remainder of this paper is organized as follows. Section II provides the details of the system model. The energy-efficient resource allocation problem is formulated in
Section III. In Section IV, we present the energy-efficient resource allocation algorithm based on an iterative Hungarian method. Simulation results and discussions are delivered in Section V. Finally, we conclude our findings in Section VI.

\section{SYSTEM MODEL}

We consider an industrial cyber physical IoT system (CPIoTS) with one central controller (CC), multiple sensors and multiple actuators as illustrated in Fig. 1. The sensors are arranged on humans, equipment, products, and buildings to collect different kinds of information, such as temperature, pressure, and position. The central controller serves as both a cloud data center and a central processor. It receives the information signals transmitted from various sensors, performs data analysis, decides what kind of operations should be performed (such as sending alerts, turning on/off a pump, moving a mechanical arm to a certain position), and then sends control signals to relevant actuators. The actuators, which are used to control the equipment in CPIoTS, receive the control signals from the central controller and perform corresponding operations. Various applications emerging in $5 \mathrm{G}$ networks can be modeled as CPIoTS, such as smart grid, smart factory and Internet of Vehicles (IoV).

The sets of sensors and actuators are denoted as $\mathcal{S}=\left\{S_{1}, S_{2}, \ldots\right.$ $\left.S_{M}\right\}$ and $\mathcal{A}=\left\{A_{1}, A_{2}, \ldots, A_{N}\right\}$, respectively. The available spectral resource is divided into multiple channels (CHs) and each channel has the same bandwidth. The set of channels is denoted as $\mathcal{C}=\left\{\mathrm{CH}_{1}, \mathrm{CH}_{2}, \ldots, \mathrm{CH}_{K}\right\}$.

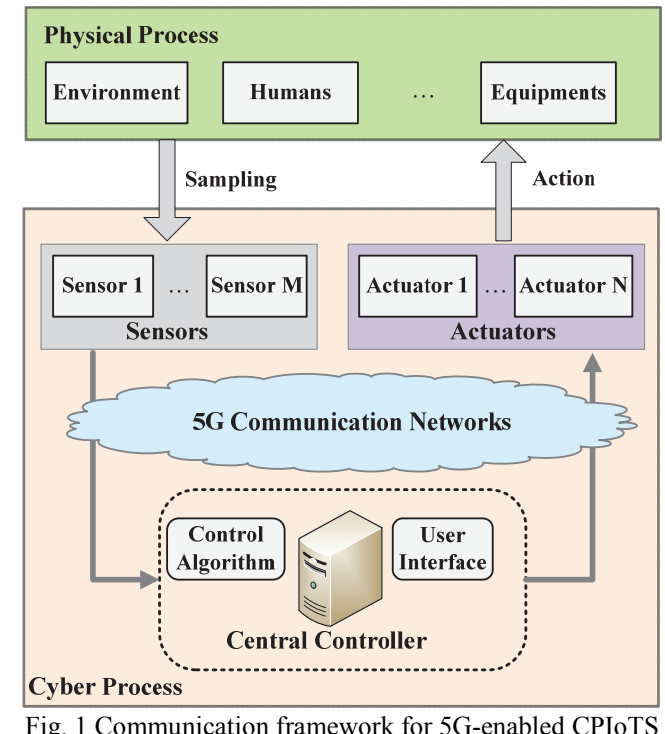

The central controller can operate either in full-duplex mode or in half-duplex mode on each channel. The central controller is common to all cellular networks, including software define network (SDN) and slice based network. The sensors and actuators can only operate in half-duplex mode due to hardware limitation. When the central controller operates in full-duplex mode on $\mathrm{CH}_{k}$, it can receive data from a sensor and transmit data to an actuator in $\mathrm{CH}_{k}$ simultaneously. In this case, the received antenna of the central controller will suffer 
self-interference from transmit antenna of itself. Fortunately, this self-interference can be suppressed by certain self-interference cancellation technology, such as analog cancellation or digital cancellation. When the central controller operates in half-duplex mode on $\mathrm{CH}_{k}, \mathrm{CH}_{k}$ can be allocated to one sensor or one actuator exclusively.

Denote $h_{i, c, k}$ and $h_{c, j, k}$ as the channel gain between sensor $S_{i}$ and $\mathrm{CC}$ on $\mathrm{CH}_{k}$, and the channel gain between $\mathrm{CC}$ and actuator $A_{j}$ on $C_{k}$ respectively, $i=1, \ldots, M, j=1, \ldots, N, k=1, \ldots, K$. Denote $g_{c, k}$ and $g_{i, j, k}$ as equivalent self-interference channel gain after interference cancellation and interference channel gain between sensor $S_{i}$ and actuator $A_{j}$ on $\mathrm{CH}_{k}$ respectively.

The achievable data rate received by actuator $A_{j}$ from central controller on $\mathrm{CH}_{k}$ can be derived as:

$$
R_{j, k}^{A}=\beta_{j, k} \log _{2}\left(1+\frac{P_{k}^{C} h_{c, j, k}}{\sum_{i=1}^{M} \alpha_{i, k} P_{i}^{S} g_{i, j, k}+\sigma_{n}}\right)
$$

where $P_{i}^{S}$ denotes the transmit power of sensor $S_{i}, P_{k}^{C}$ denotes the transmit power of $\mathrm{CC}$ on $\mathrm{CH}_{k}, W$ is channel bandwidth of each $\mathrm{CH}, \sigma_{n}$ denotes the variance of noise on each device. $\alpha_{i, k}$ and $\beta_{j, k}$ denote the channel allocation index of sensor $S_{i}$ and actuator $A_{j}$, respectively. $\alpha_{i, k}=1$ means $S_{i}$ transmitting signals to central controller on $\mathrm{CH}_{k}$, while $\alpha_{i, k}=0$ means otherwise. Similarly, $\beta_{j, k}=1$ means actuator $A_{j}$ receives signals from central controller on $\mathrm{CH}_{k}$, while $\beta_{j, k}=0$ means otherwise.

Correspondingly, the achievable rate received by central controller from sensor $S_{i}$ on $\mathrm{CH}_{k}$ can be derived as

$$
R_{i, k}^{S}=\alpha_{i, k} \log _{2}\left(1+\frac{P_{i}^{S} h_{i, c, k}}{\sum_{j=1}^{N} \beta_{j, k} P_{k}^{C} g_{c, k}+\sigma_{n}}\right)
$$

Assume channels are allocated to sensors and actuators according to the following rules: a channel can be allocated to no more than one sensor and one actuator. One sensor can only be allocated with one channel. The same assumption holds for actuators. The channel allocation constraints can be represented as $\sum_{i=1}^{M} \alpha_{i, k} \leq 1, \sum_{k=1}^{K} \alpha_{i, k} \leq 1, \sum_{j=1}^{N} \beta_{j, k} \leq 1$ and $\sum_{k=1}^{K} \beta_{j, k} \leq 1$.

Note that according to channel allocation criterions above, for each channel there are three cases of channel allocation. In the first case, one channel can be allocated to one sensor and $\mathrm{CC}$ operates in half-duplex mode (as a receiver). In the second case, one channel can be allocated to one actuator and CC operates in half-duplex mode (as a transmitter). In the third case, one channel can be allocated to one sensor and one actuator, and $\mathrm{CC}$ operates in full-duplex mode.

Considering equations (1) and (2), the energy efficiency of actuator $A_{j}$ and sensor $S_{i}$ on $C_{k}$ can be represented as

$$
\begin{aligned}
E E_{j, k}^{A} & =\frac{R_{j, k}^{A}}{\eta P_{k}^{C}+P_{c i r}} \\
E E_{i, k}^{S} & =\frac{R_{i, k}^{S}}{\eta P_{i}^{S}+P_{c i r}}
\end{aligned}
$$

where $P_{\text {cir }}$ denotes the total circuit power consumption, which mainly includes power consumption of mixer, frequency synthesizer, and digital-to-analog converter. $\eta$ is a constant depending on the power amplifier efficiency, $\eta>1$.

The utility function of this CPIoTS is defined as the sum energy efficiency of all sensors and actuators, which is represented as:

$$
U=\sum_{k=1}^{K} \sum_{i=1}^{M} E E_{i, k}^{S}+\sum_{k=1}^{K} \sum_{j=1}^{N} E E_{j, k}^{A}
$$

\section{PROBLEM FORMULATION}

In this paper, we investigate the energy-efficient resource allocation problem of sensors and actuators, aiming to maximize the utility function defined in (5) while satisfying the rate requirements of each sensor and actuator. The optimization problem can be formulated as

$$
\begin{aligned}
\text { P1: } & \max _{P_{k}^{C}, P_{i}^{S}, \alpha_{i, k}, \beta_{j, k}} U \\
\text { s.t. } & \sum_{i=1}^{M} \alpha_{i, k} \leq 1, \quad \forall k \\
& \sum_{j=1}^{N} \beta_{j, k} \leq 1, \quad \forall k \\
& \sum_{k=1}^{K} \alpha_{i, k} \leq 1, \quad \forall i \\
& \sum_{k=1}^{K} \beta_{j, k} \leq 1, \quad \forall j \\
& 0 \leq P_{k}^{C} \leq P_{\max }^{C}, \quad \forall k \\
& 0 \leq P_{i}^{S} \leq P_{\max }^{S}, \quad \forall i \\
& R_{j, k}^{A} \geq R_{\min }^{A}, \quad \forall j, \forall k \\
& R_{i, k}^{S} \geq R_{\min }^{S}, \quad \forall i, \forall k
\end{aligned}
$$

Constraints (6b), (6c), (6d) and (6e) denote the channel allocation criterions. Constraints (6f) and (6g) represent the transmit power constraints of each sensor and transmit power constraints of central controller for each actuator respectively. Constraints (6h) and (6i) denote the rate requirement of each sensor and each actuator.

To formulate the channel allocation problem in a more generalized way, we introduce the concept of virtual devices.

Definition 1: A virtual device is defined as a device which satisfies the following condition: the channel gain between a virtual device and any device is zero.

A virtual device can be a virtual sensor or a virtual actuator. Considering the definition of virtual device, it is clear that the energy efficiency of a virtual sensor or a virtual actuator is always 0 .

After the definition of virtual devices, we add $N$ virtual sensors and $M$ virtual actuators to the CPIoTS, and expand the sets of sensors and actuators as $\underline{\mathcal{S}}=\left\{S_{1}, S_{2}, \ldots S_{M}, S_{M+1}, \ldots, S_{M+N}\right\}$ and $\underline{\mathcal{A}}=\left\{A_{1}, A_{2}, \ldots, A_{N}, A_{N+1}, \ldots, A_{M+N}\right\}$, where $S_{M+1}, \ldots, S_{M+N}$ and $A_{N+1}, \ldots, A_{M+N}$ are $N$ virtual sensors and $M$ virtual actuators respectively. Correspondingly, we refer to sensors $S_{1}, \ldots, S_{M}$ in $\underline{\mathcal{S}}$ and actuators $A_{1}, \ldots, A_{N}$ in $\underline{\mathcal{A}}$ as real sensors and real actuators respectively. Note that the sensors/actuators depicted in Fig.1 are all real sensors/actuators. The virtual sensors/actuators 
introduced are not shown in Fig.1. The addition of virtual sensors and virtual actuators do not cause any changes to real devices (real sensors and real actuators) and the central controller.

After addition of virtual sensors and virtual actuators, we can regard the three channel allocation cases mentioned above as one generalized case, in which a channel is always allocated to a sensor (a real sensor or a virtual sensor) and an actuator (a real actuator or a virtual actuator). If $\mathrm{CH}_{k}$ is allocated to a real sensor and a virtual actuator, $\mathrm{CH}_{k}$ is actually allocated on a sensor only. Similarly, if $\mathrm{CH}_{k}$ is allocated to a virtual sensor and a real actuator, $\mathrm{CH}_{k}$ is actually allocated on an actuator only.

We denote a 3-dimensional channel allocation index $[\mathbf{W}]_{(M+N) \times(M+N) \times K}$ indicating the allocation of channels on sensors and actuators, in which $w_{i, j, k}=1$ when $\mathrm{CH}_{k}$ is allocated to $S_{i}$ (When $i \leq M, S_{i}$ is a real sensor. When $i>M, S_{i}$ is a virtual sensor) and $A_{j}$ (When $j \leq N, A_{j}$ is a real actuator. When $j>N, A_{j}$ is a virtual actuator); $w_{i, j, k}=0$ otherwise. Considering the definitions of $\alpha_{i, k}, \beta_{j, k}$ and $w_{i, j, k}$, the relationships among $\alpha_{i, k}, \beta_{j, k}$ and $w_{i, j, k}$ are as follows $w_{i, j, k}=\alpha_{i, k} \beta_{j, k}, \quad \alpha_{i, k}=\sum_{j=1}^{M} w_{i, j, k}$ and $\beta_{j, k}=\sum_{i=1}^{N} w_{i, j, k}$.

Then we define energy efficiency matrix as a 3-dimensional matrix $[\boldsymbol{S E E}]_{(M+N) \times(M+N) \times K}$, in which the elements $S E E_{i, j, k}$ is the sum energy efficiency of $S_{i}$ and $A_{j}$ on $\mathrm{CH}_{k}$, when $\mathrm{CH}_{k}$ is allocated on $S_{i}$ and $A_{j}$. According to (3) and (4), $S E E_{i, j, k}$ can be represented as

$$
S E E_{i, j, k}=E E_{i, k}^{S}+\left.E E_{j, k}^{A}\right|_{\alpha_{i, k}=1, \beta_{j, k}=1, \alpha_{i, k}=0, \beta_{j^{\prime}, k}=0\left(i^{\prime} \neq i, j^{\prime} \neq j\right)}
$$

Since sensor $S_{i}$ is either a real sensor $(1 \leq i \leq M)$ or a virtual sensor $(M+1 \leq i \leq M+N)$ and actuator $A_{j}$ is either a real actuator $(1 \leq j \leq N)$ or a virtual actuator $(N+1 \leq j \leq M+N), S E E_{i, j, k}$ can be calculated as follows:

$$
S E E_{i, j, k}=\left\{\begin{array}{cc}
\frac{W \log _{2}\left(1+\frac{P_{i}^{S} h_{i, c, k}}{P_{k}^{C} g_{c, k}+\sigma_{n}}\right)}{\eta P_{i}^{S}+P_{c i r}}+\frac{\log _{2}\left(1+\frac{P_{k}^{C} h_{c, j, k}}{P_{i}^{S} g_{i, j, k}+\sigma_{n}}\right)}{\eta P_{k}^{C}+P_{c i r}} \quad 1 \leq i \leq M, 1 \leq j \leq N \\
\frac{W \log _{2}\left(1+\frac{P_{i}^{S} h_{i, c, k}}{\sigma_{n}}\right)}{\eta P_{i}^{S}+P_{c i r}} & 1 \leq i \leq M, N+1 \leq j \leq M+N \\
\frac{W \log _{2}\left(1+\frac{P_{k}^{C} h_{c, j, k}}{\sigma_{n}}\right)}{\eta P_{k}^{C}+P_{c i r}} & M+1 \leq i \leq M+N, 1 \leq j \leq N \\
0 & M+1 \leq i \leq M+N, N+1 \leq j \leq M+N
\end{array}\right.
$$

From (8), if both $S_{i}$ and $A_{j}$ are real devices, $S E E_{i, j, k}$ denotes the sum energy efficiency of $S_{i}$ and $A_{j}$ in $C_{k}$. If $S_{i}$ is a real sensor and $A_{j}$ is a virtual actuator, $S E E_{i, j, k}$ equals to the energy efficiency of $S_{i}$ in $C H_{k}$. If $S_{i}$ is a virtual sensor and $A_{j}$ is a real actuator, $S E E_{i, j, k}$ equals to the energy efficiency of $A_{j}$ in $C H_{k}$. If both $S_{i}$ and $A_{j}$ are virtual devices, $S E E_{i, j, k}$ equals to 0 .

After introducing energy efficiency matrix, we can rewrite the utility function defined in (5) as follows

$$
U=\sum_{k=1}^{K} \sum_{i=1}^{M} \sum_{j=1}^{N} w_{i, j, k} S E E_{i, j, k} .
$$

Considering the relationship among $\alpha_{i, k}, \beta_{j, k}$ and $w_{i, j, k}$ and equation (9), the resource allocation problem P1 can be equivalently transformed as:

$$
\begin{aligned}
\text { P2: } & \max _{P_{k}^{C}, P_{i}^{S}, w_{i, j, k}} \sum_{i=1}^{M+N} \sum_{j=1}^{M+N} \sum_{k=1}^{K} w_{i, j, k} S E E_{i, j, k} \\
\text { s.t. } & \sum_{i=1}^{M+N} \sum_{j=1}^{M+N} w_{i, j, k} \leq 1, \quad \forall k \\
& \sum_{i=1}^{M+N} \sum_{k=1}^{K} w_{i, j, k} \leq 1, \quad \forall j \\
& \sum_{j=1}^{M+N} \sum_{k=1}^{K} w_{i, j, k} \leq 1, \quad \forall i \\
& (6 \mathrm{f}),(6 \mathrm{~g}),(6 \mathrm{~h}),(6 \mathrm{i}) .
\end{aligned}
$$

According to constraint (10b), a $\mathrm{CH}$ can only be allocated to a sensor-actuator pair. According to (10c) and (10d), a sensor or an actuator can occupy one $\mathrm{CH}$ only.

The optimization problem $\mathrm{P} 2$ is a mixed integer nonlinear programming (MINLP), which includes both continuous variables and binary variables. To reduce computational complexity, we decompose the original optimization problem into two subproblems: power allocation problem and channel assignment problem, which is a widely used solution approach for resource allocation problem. In power allocation problem, the transmit power of sensors and central controller allocated on each channel is optimized. In channel assignment problem, each channel is assigned to different sensors and actuators.

\section{ENERGY-EFFICIENT RESOURCE ALLOCATION BASED ON ITERATIVE HUNGARIAN METHOD}

\section{A. Energy-Efficient Power Allocation}

In power allocation, we aim to maximize each $S E E_{i, j, k}$ in energy efficiency matrix first. Since each $S E E_{i, j, k}$ is only dependent on $P_{k}^{C}$ and $P_{i}^{S}$, we can optimize each $S E E_{i, j, k}$ separately. The power allocation problem can be formulated as

$$
\begin{aligned}
\text { P3: } & \max _{P_{i}^{S}, P_{k}^{C}} S E E_{i, j, k} \\
& \text { s.t }(6 \mathrm{f}),(6 \mathrm{~g}),(6 \mathrm{~h}),(6 \mathrm{i})
\end{aligned}
$$

Considering the expression of $S E E_{i, j, k}$ in equation (8), we shall consider the power allocation problem P3 in different cases. Since when $M+1 \leq i \leq K, N+1 \leq j \leq K$, the $S E E_{i, j, k}$ is always 0 , there is no need for power allocation in this case and we only need to consider $S E E_{i, j, k}$ in three cases. Case 1: a real sensor $S_{i}$ and a virtual actuator $A_{j}$ are allocated on $C H_{k}, 1 \leq i \leq M$, $N+1 \leq j \leq M+N$. Case 2: a virtual sensor $S_{i}$ and a real actuator $A_{j}$ are allocated on $C_{k}, M+1 \leq i \leq M+N, 1 \leq j \leq N$. Case 3: a real sensor $S_{i}$ and a real actuator $A_{j}$ are allocated on $C H_{k}, 1 \leq i \leq M$, $1 \leq j \leq N$. In the following, we consider the solution to power allocation problem $\mathrm{P} 3$ in each case respectively.

Case 1: In this case, $\mathrm{CH}_{k}$ is allocated to a real sensor $S_{i}$ and a virtual actuator $A_{j}$. Thus only the transmit power of sensor $S_{i}$ need to be optimized. We formulate the power allocation of sensor $S_{i}$ as follows:

$$
\begin{aligned}
P 4: \max _{P_{i}^{S}} \frac{R_{i, k}^{S}\left(P_{i}^{S}\right)}{\eta P_{i}^{S}+P_{c i r}} \\
\text { s.t } 0 \leq P_{i}^{S} \leq P_{\text {max }}^{S}
\end{aligned}
$$




$$
R_{i, k}^{S}\left(P_{i}^{S}\right) \geq R_{\min }^{S}
$$

where $R_{i, k}^{S}\left(P_{i}^{S}\right)$ denotes the transmit rate of sensor $S_{i}$. In this case, $R_{i, k}^{S}\left(P_{i}^{S}\right)$ can be derived as $R_{i, k}^{S}\left(P_{i}^{S}\right)=W \log _{2}\left(1+\frac{P_{i}^{S} h_{i, c, k}}{\sigma_{n}}\right)$.

Obviously, the optimization problem $\mathrm{P} 4$ only includes one variable $P_{i}^{s}$. The maximum power constraint (12b) is linear and the minimum rate requirement constraint (12c) can be converted into a linear constraint. However, since the objective function is a nonlinear fraction function, problem P4 is obviously not convex [18]. Thus, we cannot solve the optimization problem P4 directly. In the following, we try to solve $\mathrm{P} 4$ by transforming the nonconvex problem in fractional form to an equivalent convex problem. First, we define the optimum solution of $\mathrm{P} 4$ as

$$
q_{i, k}^{S^{*}}=\max _{P_{i}^{S}} \frac{R_{i, k}^{S}\left(P_{i}^{S^{*}}\right)}{\eta P_{i}^{S^{*}}+P_{\text {cir }}}
$$

where $P_{i}^{S^{*}}$ is the solution of power allocation problem P4. According to [19], we have Theorem 1.

Theorem 1: $q_{i, k}^{s^{*}}$ is achieved if and only if

$$
\max _{P_{i}^{S}} R_{i, k}^{S}\left(P_{i}^{S}\right)-q_{i, k}^{S^{*}}\left(\eta P_{i}^{S}+P_{c i r}\right)=R_{i, k}^{S}\left(P_{i}^{S^{*}}\right)-q_{i, k}^{S^{*}}\left(\eta P_{i}^{S^{*}}+P_{c i r}\right)=0
$$

According to Theorem 1, we can transform optimization problem $\mathrm{P} 4$ as follows

$$
\begin{aligned}
\text { P4.1: } \max _{P_{i}^{S}} R_{i, k}^{S}\left(P_{i}^{S}\right)-q_{i, k}^{S *}\left(\eta P_{i}^{S}+P_{c i r}\right) \\
\text { s.t } 0 \leq P_{i}^{S} \leq P_{\max }^{S} \\
P_{i}^{S} \geq B_{i, c, k}
\end{aligned}
$$

where $B_{i, c, k}=\sigma_{n}\left(2^{R_{\min }^{S} / W}-1\right) / h_{i, c, k}$. Constraint (14c) is equivalent to constraint (12c). Since the objective function of P4.1 is convex and both constraint (14b) and constraint (14c) are linear, optimization problem $\mathrm{P} 4.1$ is a convex problem and has the same solution with optimization P4. However, since the value of $q_{i, k}^{s^{*}}$ is dependent on the solution of problem P4, $q_{i, k}^{S^{*}}$ is unknown during solving this problem. To solve problem P4.1, we consider the following optimization problem

$$
\begin{gathered}
P 4.2: \max _{P_{i}^{S}} R_{i, k}^{S}\left(P_{i}^{S}\right)-q_{i, k}^{S}\left(\eta P_{i}^{S}+P_{c i r}\right) \\
\text { s.t }(14 \mathrm{~b}),(14 \mathrm{c})
\end{gathered}
$$

We propose an iterative algorithm based on Dinkelbach's algorithm for optimization problem P4.1 by solving problem P4.2 iteratively [20]. Particularly, we initialize the algorithm by assuming each sensor transmits with its maximum power $P_{\max }^{S}$. In this case, the energy efficiency of each sensor $q_{i, k}^{S}$ can be calculated. In each iteration, convex problem P4.2 is solved and the value of $q_{i, k}^{S}$ is updated until convergence. The details of the algorithm are presented in Algorithm 1.

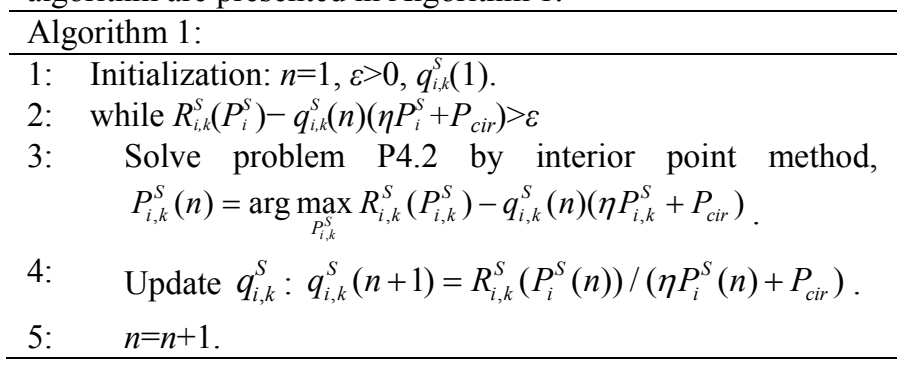

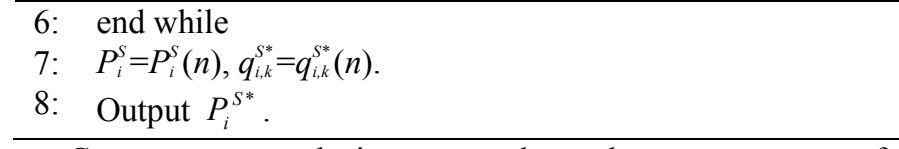

Convergence analysis: we analyze the convergence of Algorithm 1 in the following. First, we shall prove that $q_{i, k}^{s}$ is non-decreasing after each iteration.

Since $P_{i}^{S}(n)=\arg \max _{P_{i}^{S}} R_{i, k}^{S}\left(P_{i}^{S}\right)-q_{i, k}^{S}(n)\left(\eta P_{i}^{S}+P_{c i r}\right)$, then we have

$$
\begin{aligned}
& R_{i, k}^{S}\left(P_{i}^{S}(n)\right)-q_{i, k}^{S}(n)\left(\eta P_{i}^{S}(n)+P_{c i r}\right) \\
& \geq R_{i, k}^{S}\left(P_{i}^{S}(n-1)\right)-q_{i, k}^{S}(n)\left(\eta P_{i}^{S}(n-1)+P_{c i r}\right) . \\
& =0
\end{aligned}
$$

Considering $q_{i, k}^{S}(n+1)=\frac{R_{i, k}^{S}\left(P_{i}^{S}(n)\right)}{\eta P_{i}^{S}(n)+P_{c i r}}$, then we have

$$
\begin{aligned}
& R_{i, k}^{S}\left(P_{i}^{S}(n)\right)-q_{i, k}^{S}(n)\left(\eta P_{i}^{S}(n)+P_{\text {cir }}\right) \\
& =q_{i, k}^{S}(n+1)\left(\eta P_{i}^{S}(n)+P_{c i r}\right)-q_{i, k}^{S}(n)\left(\eta P_{i}^{S}(n)+P_{c i r}\right) \\
& \geq 0
\end{aligned}
$$

Since $\eta P_{i}^{S}(n)+P_{c i r}>0$, we have $q_{i, k}^{S}(n+1) \geq q_{i, k}^{S}(n)$.

Also, we shall prove that $\lim _{n \rightarrow \infty} q_{i, k}^{S}(n)=q_{i, k}^{S^{*}}$. Suppose $\lim _{n \rightarrow \infty} q_{i, k}^{S}(n)=\tilde{q}_{i, k}^{S} \neq q_{i, k}^{S^{*}}$, we must have $\tilde{q}_{i, k}^{S}<q_{i, k}^{S^{*}}$. Define function $\quad F(q) \quad$ as $\quad F(q)=\max _{P_{i}^{S}} R_{i, k}^{S}\left(P_{i}^{S}\right)-q\left(\eta P_{i}^{S}+P_{\text {cir }}\right)$. According to [20], function $F(q)$ is a monotonic decreasing function. According to the terminal condition of Algorithm 1, we have $F\left(\tilde{q}_{i, k}^{S}\right)=0$. On the other hand, we have $F\left(q_{i, k}^{S^{*}}\right)=0$ according to Theorem 1 . Thus this contradicts with the fact that $\tilde{q}_{i, k}^{S}<q_{i, k}^{S^{*}}$. Hence it follows that $\lim _{n \rightarrow \infty} F\left(q_{i, k}^{S}(n)\right)=F\left(q_{i, k}^{S^{*}}\right)$ and $\lim _{n \rightarrow \infty} q_{i, k}^{S}(n)=q_{i, k}^{S *}$.

Case 2: In this case, channel $C H_{k}$ is assigned to a virtual $S_{i}$ and a real actuator $A_{j}$ and only the transmit power of central controller on $\mathrm{CH}_{k} P_{k}^{C}$ needs to be optimized. The power allocation can be formulated as

$$
\begin{gathered}
P 5: \max _{P_{k}^{C}} \frac{R_{j, k}^{A}}{\eta P_{k}^{C}+P_{c i r}} \\
\text { s.t } 0 \leq P_{k}^{C} \leq P_{\max }^{C} \\
R_{j, k}^{A} \geq R_{\min }^{A}
\end{gathered}
$$

where $R_{j, k}^{4}$ denotes the achievable rate of actuator $A_{j}$. In this case $R_{j, k}^{A}$ can be derived as $R_{j, k}^{A}=W \log _{2}\left(1+\frac{P_{k}^{C} h_{c, j, k}}{\sigma_{n}}\right)$.

The formation of problem P5 is similar to that of problem P4. Thus the solution is similar to the solution of problem P4. The details of the solution are omitted due to space limitation.

Case 3: In this case, $C_{k}$ is allocated to a real sensor $S_{i}$ and a real actuator $A_{j}$ and the power allocation problem of $S_{i}$ and $A_{j}$ on $\mathrm{CH}_{k}$ can be formulated as follows

$$
\begin{aligned}
P 6: \max _{P_{i}^{S}, P_{k}^{C}} & \frac{R_{i, k}^{S}}{\eta P_{i}^{S}+P_{c i r}}+\frac{R_{j, k}^{A}}{\eta P_{k}^{C}+P_{c i r}} \\
\text { s.t } 0 & \leq P_{i}^{S} \leq P_{\max }^{S}
\end{aligned}
$$




$$
\begin{gathered}
0 \leq P_{k}^{C} \leq P_{\max }^{C} \\
R_{j, k}^{A} \geq R_{\min }^{A} \\
R_{i, k}^{S} \geq R_{\min }^{S}
\end{gathered}
$$

where $R_{i, k}^{S}$ and $R_{j, k}^{4}$ denote the achievable rate of sensor $S_{i}$ and actuator $A_{j}$ respectively. In this case, $R_{i, k}^{s}$ and $R_{j, k}^{A}$ can be derived

$$
\text { as } \quad R_{i, k}^{S}=W \log _{2}\left(1+\frac{P_{i}^{S} h_{i, c, k}}{P_{k}^{C} g_{c, k}+\sigma_{n}}\right) \quad, \quad \text { and }
$$$$
R_{j, k}^{A}=W \log _{2}\left(1+\frac{P_{k}^{C} h_{c, j, k}}{P_{i}^{S} g_{i, j, k}+\sigma_{n}}\right) .
$$

The energy-efficient power allocation problem P6 is modeled as a sum-of-ratios problem (SoRP). The objective function is a summation of two terms and each term has the form of concave-convex fractional function. Thus the objective function is neither a pseudo-concave nor a quasi-concave function.

To tackle problem $\mathrm{P} 6$, we propose a game theoretical approach to solve the power allocation problem of both sensor $S_{i}$ and actuator $A_{j}$. During the solution of optimization problem P6, we formulate a non-cooperative game with two players: $S_{i}$ and $A_{j}$. Sensor $S_{i}$ and actuator $A_{j}$ are regarded as rational and selfish players who will choose their strategies depending on their partner's decision. In this game, the strategies set of each player regards its feasible region of transmit power and the payoff regards its corresponding energy efficiency. Since each player only cares about its own payoff, if player $S_{i}$ knows the transmit power of player $A_{j}$, player $S_{i}$ will choose its transmit power according to the solution to the following power allocation problem:

$$
\begin{gathered}
\text { P6.1: } \max _{P_{i}^{S}} \frac{R_{i, k}^{S}}{\eta P_{i}^{S}+P_{\text {cir }}} \\
\text { s.t } 0 \leq P_{i}^{S} \leq P_{\max }^{S} \\
P_{i}^{S} \geq O_{i, b, k}
\end{gathered}
$$

where $O_{i, k}=\left(P_{k}^{C} g_{c, k}+\sigma_{n}\right)\left(2^{R_{\min }^{S} / W}-1\right) / h_{i, c, k}$. Constraint $(18 \mathrm{c})$ is an equivalent transform of constraint (17e).

Similarly, player $A_{j}$ chooses its transmit power according to the solution to the following power allocation problem

$$
\begin{gathered}
\text { P6.2: } \max _{P_{k}^{C}} \frac{R_{j, k}^{A}}{\eta P_{k}^{C}+P_{c i r}} \\
\text { s.t } 0 \leq P_{k}^{C} \leq P_{\max }^{C} \\
P_{k}^{C} \geq Q_{i, j, k}
\end{gathered}
$$

where $Q_{i, j, k}=\left(P_{i}^{S} g_{i, j, k}+\sigma_{n}\right)\left(2^{R_{\min }^{A} / W}-1\right) / h_{c, j, k}$. Constraint $(19 \mathrm{c})$ is derived by equivalent transform of constraint (17d).

Note that the two optimization problems P6.1 and P6.2 are inter-correlated through the interference terms. In other words, the solution of problem P6.1 is dependent on the transmit power $P_{k}^{C}$. Also, the solution of P6.2 is dependent on the transmit power $P_{i}^{S}$. To explore the characteristic of this game, we introduce Lemma 1.

Lemma 1: There exists at least one Nash equilibrium of this non-cooperative game.
Proof: According to [21], a Nash equilibrium exists if the utility function is continuous and quasi-concave and the set of strategies is a nonempty compact convex subset of Euclidean space. Considering energy efficiency of sensor $S_{i}$ defined in (2), the numerator $R_{i, k}^{S}$ is a concave function of $P_{i}^{S}$ and the denominator is an affine function of $P_{i}^{s}$. Therefore, energy efficiency $E E_{i, k}^{s}$ is quasi-concave. The set of strategies of sensor $S_{i}\left(0, P_{\max }^{S}\right)$ is a nonempty compact convex subset of the Euclidean space. Similarly, it is easy to prove that the above conditions also hold for the player $A_{j}$. Therefore, a Nash equilibrium exists in this non-cooperative game.

Similar to the solution of problem P4 in case 1, the energy efficiency optimization problems P6.1 and P6.2 are modeled as fractional programming and can be solved by Algorithm 1 . Then we propose an iterative algorithm to achieve the Nash equilibrium. In each iteration, sensor $S_{i}$ and actuator $A_{j}$ optimize their transmit power using Algorithm 1 according to their partner's transmit power. After finite iterations, the Nash equilibrium of this repeated game can be achieved. The iterative algorithm is detailed in Algorithm 2.

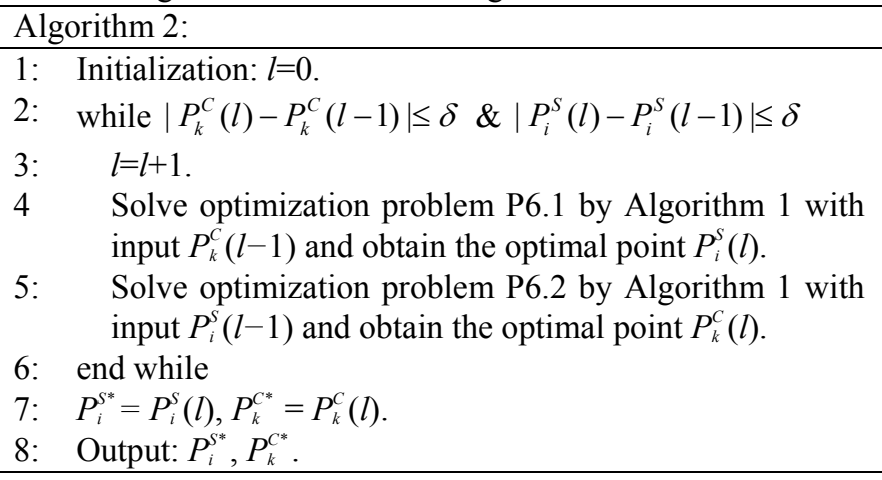

To prove that the result obtained in Algorithm 2 is the Nash equilibrium, we shall present Lemma 2.

Lemma 2: The power allocation strategy set $\left(P_{i}^{S^{*}}, P_{k}^{C^{*}}\right)$ obtained by the iterative algorithm constitutes a Nash equilibrium. None individual device is able to unilaterally get better performance by deviating from Nash equilibrium.

Proof: Suppose the strategy $P_{i}^{s^{*}}$ obtained by the Algorithm 2 is not the Nash equilibrium, which means $S_{i}$ can choose the Nash equilibrium $\hat{P}_{i}^{S}$ to obtain the maximum energy efficiency when $A_{j}$ choose the strategy $P_{k}^{C^{*}}$. However, this contradicts with the fact that $P_{i}^{S^{*}}$ is a solution of problem P6.1. A similar proof also holds for $P_{k}^{c^{*}}$. Thus we can conclude that the iterative algorithm can obtain a Nash equilibrium.

\section{B. Channel assignment}

In the power allocation step, each $S E E_{i, j, k}$ is optimized. Then we consider the channel assignment problem, which is formulated as follows

$$
\begin{aligned}
\text { P7: } & \max _{w_{i, j, k}} \sum_{i=1}^{M+N} \sum_{j=1}^{M+N} \sum_{k=1}^{K} w_{i, j, k} S E E_{i, j, k}^{*} \\
\text { s.t. } & \sum_{i=1}^{M+N} \sum_{j=1}^{M+N} w_{i, j, k} \leq 1, \quad \forall k \\
& \sum_{i=1}^{M+N} \sum_{k=1}^{K} w_{i, j, k} \leq 1, \quad \forall j
\end{aligned}
$$




$$
\sum_{k=1}^{K} \sum_{j=1}^{M+N} w_{i, j, k} \leq 1, \quad \forall i
$$

where $S E E_{i, j, k}^{*}$ is the optimal value of $S E E_{i, j, k}$ derived in the

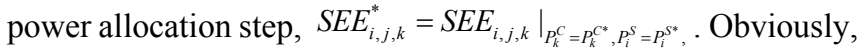
only binary variables are involved in this problem. The channel assignment problem (20) can be modeled as a 3-dimensional one-to-one matching problem, which is known as an NP-hard problem.

\section{1) 3-Dimensional Matching}

We first present the definition of 3-dimensional matching.

Definition 2: Given three disjoint sets $\mathcal{M}, \mathcal{N}$ and $\mathcal{W}$, a 3-dimensional one-to-one matching $\mu$ is defined as a mapping from $\mathcal{M} \cup \mathcal{N} \cup \mathcal{W}$ to subsets of $\mathcal{M} \cup \mathcal{N} \cup \mathcal{W}$, such that for $m \in$ $\mathcal{M}, \mu(m)=(n, w), n \in \mathcal{N}, w \in \mathcal{W}$, for $n \in \mathcal{N}, \mu(n)=(m, w), m \in$ $\mathcal{M}, w \in \mathcal{W}$, and for $w \in \mathcal{W}, \mu(w)=(m, n), m \in \mathcal{M}, n \in \mathcal{N}$.

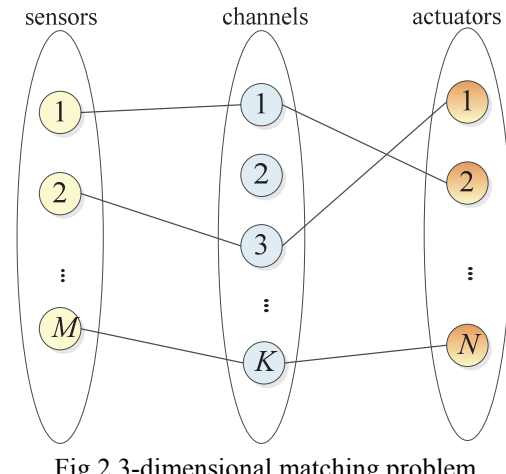

According to the definition of 3-dimensional matching, the channel assignment for CPIoTS can be regarded as a 3-dimensional matching among three sets $(\mathcal{C}, \underline{\mathcal{I}}$ and $\underline{\mathcal{R}})$. The payoff of matching triple $\left(S_{i}, A_{j}, C_{k}\right)$ denotes the sum energy efficiency of $S_{i}$ and $A_{j}$ on $C_{k}$. Our goal is to find the optimal matching to maximize the overall payoff

The 3-dimensional matching can be illustrated in another way. It is well known that 2-dimensional matching problem is also called task assignment problem, in which a task should be assigned to one agent with certain payoff [22]. Correspondingly, a 3-dimensional matching problem can be considered as an enhanced task assignment problem in which a task $(\mathrm{CH})$ should be accomplished by a pair of agents (one sensor and one actuator) corporately. Different agent pairs have different payoffs after completing one task. The goal of a 3-dimensional matching is to find the optimal assignment by which the overall payoff can be maximized.

\section{2) Iterative Hungarian Method with Virtual Devices}

Inspired by the fact that the 2-dimensional matching problem (task assignment problem) can be solved by Hungarian algorithm [23], we propose an iterative algorithm based on Hungarian algorithm to tackle this 3-dimensional problem, which is regarded as Iterative Hungarian Method with Virtual Devices (IHM-VD). In each iteration, a 2-dimensional matching is implemented between one set and the set of matching pairs of other two sets. Then the 3-dimensional allocation matrix $\mathrm{W}$ is updated in each iteration. The algorithm continues until a stable allocation is achieved.

We first consider a random channel allocation matrix $\mathrm{W}_{0}$ as initial allocation, which meets the constraints in (20). Then we consider the 2-dimensional matching between the set of sensors and the set of matched actuator-channel pairs. Denote the set of matched actuator-channel pairs as $\mathcal{X}_{1}, \quad \mathcal{X}_{1}=\left\{(j, k) \mid w_{i, j, k}=1\right.$, $\left.w_{i, j, k} \in \mathrm{W}_{0}\right\}$. We use index $l$ to indicate each actuator-channel pair, $1<l<\min (M+N, K)$. $S E E_{i, l}$ denotes the sum energy efficiency when sensor $S_{i}$ matches the $l$ th actuator-channel pair. Then the original 3-dimensional matching problem is reduced to a 2-dimensional matching problem between $\underline{\mathcal{T}}$ (the set of sensors) and $\mathcal{X}_{1}$ (the set of matched actuator-channel pairs). A 2-dimensional index matrix $[\mathrm{X}]_{(M+N) \times \min (M+N, K)}$ denotes the matching result between sensors and actuator-channel pairs, where $x_{i, l}=1$, if sensor $S_{i}$ matches the $l$ th actuator-channel pair; $x_{i, l}=0$, otherwise. The sub-optimization problem can be formulated as:

$$
\begin{aligned}
\text { P7.1 } & \max _{X} \sum_{i=1}^{M+N} \sum_{l=1}^{\min (M+N, K)} x_{i, l} S E E_{i, l} \\
\text { s.t. } & \sum_{i=1}^{M+N} x_{i, l} \leq 1, \quad \sum_{l=1}^{\min (M+N, K)} x_{i, l} \leq 1
\end{aligned}
$$

The optimal solution $\mathrm{X}^{*}$ to 2-dimensional matching problem P7.1 can be obtained using Hungarian algorithm. By joint considering $\mathrm{X}^{*}$ and $\mathcal{X}_{1}$, we can update the 3-dimensional allocation matrix $\mathrm{W}_{1}=\left[\mathrm{X}^{*}, \mathcal{X}_{1}\right]$.

Then we consider the 2-dimensional matching between the set of channels and the set of matched sensor-actuator pairs. Denote the set of matched sensor-actuator pairs as $\mathcal{X}_{2}, \mathcal{X}_{2}=\{(i$, $\left.j) \mid w_{i, j, k}=1, w_{i, j, k} \in \mathrm{W}_{1}\right\}$. And we use index $r$ to indicate each sensor-actuator pair, $1<r<M+N$. $S E E_{r, k}$ denotes the sum energy efficiency when $\mathrm{CH}_{k}$ matches the $r$ th sensor-actuator pair. Also, we consider a 2-dimensional matching between $\mathcal{C}$ (the set of $\mathrm{CHs}$ ) and $\mathcal{X}_{2}$ (the set of matched sensor-actuator pairs). Denote a 2-dimensional index matrix $[\mathrm{Y}]_{K_{\times}(M+N)}$ as the matching result between sensor-actuator pairs and $\mathrm{CHs}$, where $y_{r, k}=1$, if the $r$ th sensor-actuator pair matches $\mathrm{CH}_{k} ; y_{r, k}=0$, otherwise. The sub-optimization problem can be formulated as:

$$
\begin{aligned}
\text { P7.2 } & \max _{Y} \sum_{k=1}^{K} \sum_{r=1}^{M+N} y_{r, k} S E E_{r, k} \\
\text { s.t. } & \sum_{r=1}^{M+N} y_{r, k} \leq 1, \sum_{k=1}^{K} y_{r, k} \leq 1
\end{aligned}
$$

The optimal solution $Y^{*}$ to 2-dimensional matching problem P7.2 can be solved by Hungarian algorithm. Then we can update the 3-dimensional allocation matrix $\mathrm{W}_{2}=\left[Y^{*}, \mathcal{X}_{2}\right]$.

Then we further consider the 2-dimensional matching between the set of actuators and the set of matched sensor-channel pairs. Denote the set of matched sensor-channel pairs as $\mathcal{X}_{3}, \mathcal{X}_{3}=\left\{(i, k) \mid w_{i, j, k}=1, w_{i, j, k} \in \mathrm{W}_{2}\right\}$. And we use index $d$ to indicate each sensor-channel pair, $1<d<K$. $S E E_{d, j}$ denotes the sum energy efficiency when actuator $A_{j}$ matches the $d$ th sensor-channel pair. Then we consider a 2-dimensional 
matching between $\underline{\mathcal{R}}$ (the set of actuators) and $\mathcal{X}_{3}$ (the set of matched sensor-channel pairs). Denote a 2-dimensional index matrix $[Z]_{(M+N) \times \min (M+N, K)}$ as the matching result between actuators and sensor-channel pairs, where $z_{d, j}=1$ if the $d$ th sensor-channel pair matches $A_{j} ; z_{d, j}=0$, otherwise. The sub-optimization problem can be formulated as:

$$
\begin{aligned}
\text { P7.3 } & \max _{Z} \sum_{j=1}^{M+N} \sum_{d=1}^{\min (M+N, K)} z_{d, j} S E E_{d, j} \\
\text { s.t. } & \sum_{d=1}^{\min (M+N, K)} z_{d, j} \leq 1, \sum_{j=1}^{M+N} z_{d, j} \leq 1
\end{aligned}
$$

The optimal solution $Z^{*}$ to 2-dimensional matching problem P7.3 can be solved by Hungarian algorithm. Then the 3-dimensional allocation matrix can be updated as $\mathrm{W}_{3}=\left[Z^{*}, \mathcal{X}_{3}\right]$.

In each iteration, the three 2-dimensional matching problems (P7.1, P7.2 and P7.3) are solved sequentially and after each 2-dimensional matching the 3-dimensional allocation matrix is updated as follows: $\mathrm{W}_{0} \rightarrow \mathrm{W}_{1} \rightarrow \mathrm{W}_{2} \rightarrow \mathrm{W}_{3} \rightarrow \mathrm{W}_{4} \rightarrow \ldots$. The iteration continues until the 3-dimensional allocation matrix remains stable or the maximum iteration number is achieved. The details of Iterative Hungarian method with Virtual Devices (IHM-VD) are listed in Algorithm 3.

Algorithm 3 Iterative Hungarian Method with Virtual Devices

Input: $[S E E]_{(M+N) \times(M+N) \times K}$
Output: $\mathrm{W}$
1: $\quad$ Initialization $\mathrm{W}_{0}, n=0, N_{\max }$.
2: $\quad$ Repeat
3: $\quad n=n+1$.

4: $\quad$ Obtain matched actuator-channel pairs set $\mathcal{X}_{1}$ and 2-dimensional energy efficiency $S E E_{i, l}$ with $i$ th sensor and $l$ th actuator-channel pair.

5: $\quad$ Solve 2-dimensional matching problem P7.1 using Hungarian method and get optimal allocation $\mathrm{X}^{*}$.

6: $\quad$ Allocation result update, $\mathrm{W}_{n}=\left[\mathrm{X}^{*}, \mathcal{X}_{1}\right]$.

7: $\quad n=n+1$.

8: $\quad$ Obtain matched sensor-actuator pairs set $\mathcal{X}_{2}$ and 2-dimensional energy efficiency $S E E_{r, k}$ with $k$ th channel and $r$ th sensor-actuator pair.

9: $\quad$ Solve 2-dimensional matching problem P7.2 using Hungarian method and get optimal allocation $\mathrm{Y}^{*}$.

10: Allocation result update, $\mathrm{W}_{n}=\left[\mathrm{Y}^{*}, \mathcal{X}_{2}\right]$.

11: $n=n+1$

12: Obtain matched sensor-channel pairs set $\mathcal{X}_{3}$ and 2-dimensional energy efficiency $S E E_{d, j}$ with $j$ th actuators and $d$ th sensor-channel pair.

13: Solve 2-dimensional matching problem P7.3 using Hungarian method and get optimal allocation $Z^{*}$.

14: $\quad$ Allocation result update, $\mathrm{W}_{n}=\left[\mathrm{Z}^{*}, \mathcal{X}_{3}\right]$.

15: Until the allocation result remains stable or $n=N_{\max }$ 16: return $\mathrm{W}^{*}=\mathrm{W}_{n}$.

As Hungarian algorithm can always achieve an optimal matching for a 2-dimensional matching, in each iteration the utility is either increased or unchanged after each 2-dimensional matching. Also, it is clear that the utility has an upper bound. Thus, we can conclude that the algorithm can achieve convergence after finite iterations. If the utility is unchanged in one iteration, the algorithm terminates and the convergence is achieved.

Assuming that the algorithm achieves convergence after $I$ iterations, the number of 2-dimensional matching can be derived as $3 I$. Since the computational complexity of 2-dimensional matching can be regarded as $\mathcal{O}\left(K^{3}\right)$ approximately, the computational complexity of IHM-VD is approximate to $\mathcal{O}\left(3 I K^{3}\right)$.

\section{SIMULATION RESULTS}

In this section, we compare the performance of the proposed IHM-VD with the following benchmarks: greedy algorithm (GA), exhaustive search (ES) and two-sided Hungarian algorithm (TSH). We also perform the simulation of a half-duplex resource allocation algorithm (HDRA) and Iterative Hungarian Method without virtual users (IHM) [23]. The details of the benchmark algorithms are as follows.

In the $\mathrm{GA}$, each $\mathrm{CH}$ selects a sensor-actuator pair with the best sum energy efficiency for itself sequentially and exclusively. Note that $\mathrm{CH}$ can also select one sensor or one actuator according to energy efficiency maximization criterion.

ES provides an optimal solution of the 3-dimensional matching problem, and the computational complexity is $\mathcal{O}(K ! K !)$. To reduce the complexity, we propose a low complexity algorithm for ES to achieve the optimal solution, which is implemented in two steps. In the first step, each possible sensor-actuator matching solution is obtained by exhaustively searching, which complexity is $\mathcal{O}(K !)$. In the second step, the optimal matching between sensor-actuator pairs and channels is accomplished by Hungarian method with complexity $\mathcal{O}\left(K^{3}\right)$. Thus the computational complexity of this low complexity exhaustive search is $\mathcal{O}\left(K^{3} K !\right)$.

In $\mathrm{TSH}$, the 3-dimensional matching problem (sensor-actuator-channel) is decoupled as two 2-dimensional matching problems (sensor-channel and actuator-channel). In other words, the channels for sensors and those for actuators are allocated separately. Each 2-dimensional matching problem is solved by Hungarian algorithm with complexity $\mathcal{O}\left(K^{3}\right)$ [22]. Thus the computational complexity of TSH is $\mathcal{O}\left(2 K^{3}\right)$. Note that two-sided Hungarian algorithm ignores the peer effects between different sensor-actuator pairs.

In IHM, no virtual devices are added in CPIoTS, and a CH is always allocated to both a real sensor and a real actuator using iterative Hungarian method. In HDRA, the central controller operates in half-duplex mode and a $\mathrm{CH}$ can be only allocated to a sensor or an actuator.

In the simulation, we assume that the channel gain contains the normalized small-scale fading and distance based large-scale pathloss, $h=d^{-\alpha} \bar{h}$, where $d$ denotes the distance 
between the transmitter and the receiver, $\alpha$ denotes the pathloss factor, $\bar{h}$ denotes the normalized Rayleigh fading. The simulation parameters are listed in Table I.

Table I Simulation Parameters

\begin{tabular}{ll}
\hline Simulation Parameters & Value \\
\hline Pathloss factor $(\alpha)$ & 4 \\
Distance between any sensor/actuator to CC $(d)$ & $10-50 \mathrm{~m}$ \\
Interference cancellation coefficient $(\eta)$ & $-60 \mathrm{~dB}$ \\
Maximum transmit power of sensors $\left(P_{\max }^{S}\right)$ & $25 \mathrm{dBm}$ \\
Maximum transmit power of CC on each CH $\left(P_{\max }^{C}\right)$ & $30 \mathrm{dBm}$ \\
Noise Power $\left(\sigma_{n}\right)$ & $-114 \mathrm{dBm}$ \\
\hline & \\
\hline
\end{tabular}

Fig. 3 Sum energy efficiency with different number of channels

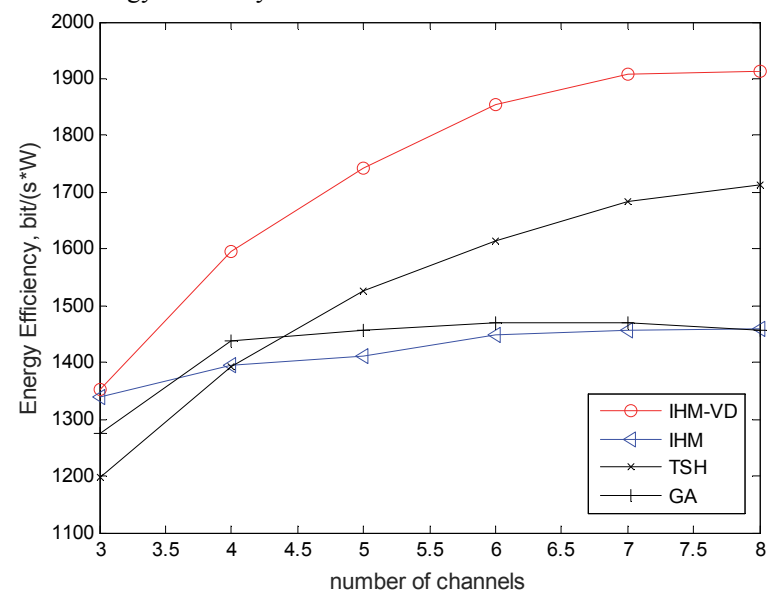

Fig. 4 Sum energy efficiency with different number of channels

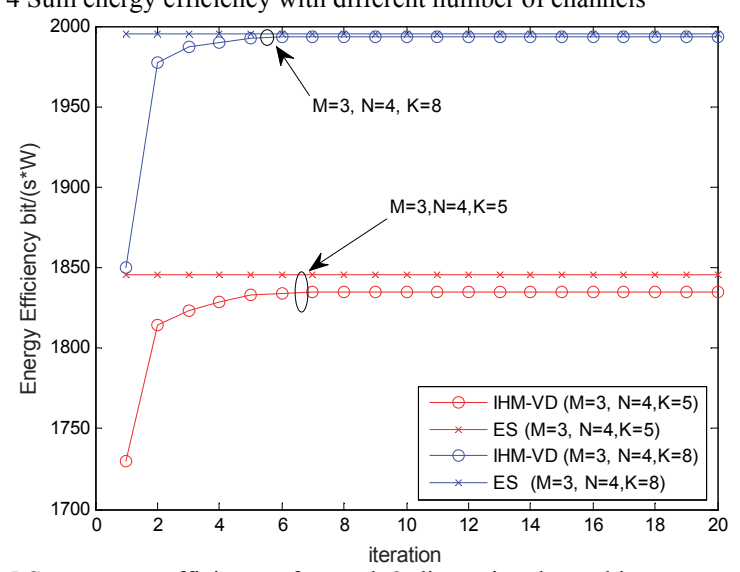

Fig. 5 Sum energy efficiency after each 2 -dimensional matching
Fig. 3 plots the sum energy efficiency versus the number of channels for IHM-VD, IHM and HDRA, considering $M=4$, $N=4$. As illustrated in Fig. 3, when the number of channels $\mathrm{K}$ is less 4 , the performance of our proposed algorithm IHM-VD is slightly better than IHM, but is much better than that of half-duplex resource allocation. This implies that when the number of channels is less than the number of sensors $M$ or that of actuators $N$, each channel is more likely shared by a real sensor and a real actuator.

When the number of channels is larger than 8 , our algorithm outperforms the IHM significantly and achieve almost the same performance with the HDRA. This is due to the fact when the number of channels is equal to or larger than the sum of the number of sensors and that of actuators, each sensor or actuator can be allocated with one dedicated channel and do not need to share its channel with another device.

To indicate that our proposed algorithm performs better than the other state-of-the-art algorithms, Fig. 4 plots the sum energy efficiency versus the number of channels for IHM-VD, TSH and GA. From Fig. 4, we can observe that our proposed algorithm achieves the best performance among three algorithms. The performance of our proposed algorithm and TTMA increases with the number of channels, while the performance of greedy algorithm nearly remains the same when the number of channels is larger than 4 .

To further illustrate the convergence of our proposed algorithm (IHM-VD), Fig. 5 shows the sum energy efficiency after each 2-dimensional matching, as well as the performance of exhaustive search (ES). We can observe that after about 6 times of 2-dimensional matching, our algorithm converges to a stable solution. Also, the performance of our proposed algorithm is close to that of exhaustive searching, but the computational complexity is much less.

\section{CONCLUSION}

In this paper, we presented a communication network framework based on 5G technology for CPIoTSs supporting multiple sensors, multiple actuators and a central controller with the ability of full-duplex communication. Based on this framework, we proposed an energy-efficient resource allocation algorithm, in which the power allocation and channel allocation was optimized separately. First, a power allocation algorithm was proposed based on Dinkelbach's algorithm and game theory to achieve the maximum sum energy efficiency for each possible channel allocation result. Then, a channel allocation algorithm named iterative Hungarian method with virtual devices was proposed. Simulation results showed that the proposed resource allocation algorithm IHM-VD outperformed the state-of-art benchmarks and could nearly achieve the performance of the exhaustive searching method. 


\section{REFERENCES}

[1] O. Givehchi, K. Landsdorf, P. Simoens, A. Colombo, "Interoperability for industrial cyber-physical systems: an approach for legacy systems", IEEE Transactions on Industrial Informatics, vol. PP, no. 99, Aug. 2017.

[2] Gartner Newsroom, "Press release: Gartner says 6.4 billion connected 'things' will be in use in 2016, up 30 percent from 2015" (November 2015) http://www.gartner.com/newsroom/id/3165317.

[3] S. Al-Rubaye, E. Kadhum, Q. Ni, and A. Anpalagan, "Industrial Internet of Things driven by SDN platform for smart grid resiliency," IEEE Internet of Things Journal, vol. PP, no.99, pp. 1-11, Aug. 2017.

[4] R. Atat, L. Liu, H. Chen, J. Wu, H. Li, and Y. Yi, "Enabling cyber-physical communication in $5 \mathrm{G}$ cellular networks: challenges, spatial spectrum sensing, and cyber-security", IET Cyber-Physical Systems: Theory \& Applications, vol.2, no.1, pp.49-54, 2017.

[5] M. Agiwal, A. Roy, and N. Saxena, "Next generation 5G wireless networks: a comprehensive survey," IEEE Communications Survey and Tutorial, vol.18, no.3, pp.1617-1655, Feb. 2016.

[6] J. Zhao, Y. Liu, K. K. Chai, and M. Elkashlan, "Matching with peer effects for context-aware resource allocation in D2D communications", IEEE Communications Letters, vol. 21, no. 4, pp. 837-840, Apr. 2017.

[7] J. Zhao, Y. Liu, K. K. Chai, and M. Elkashlan, "Many-to-many matching with externalities for device-to-device communications," IEEE Wireless Communications Letters, vol. 6, no. 1, pp.138-141, Feb. 2017.

[8] D. Kim, H. Lee, and D. Hong, "A Survey of in-band full-duplex transmission: From the perspective of PHY and MAC layers", IEEE Communications Survey and Tutorials, vol.12, no.4, pp. 2017-2016, 2015.

[9] X. Zhang, W. Cheng, and H. Zhang, Full-duplex transmission in PHY and MAC layers for 5G mobile wireless networks," IEEE Wireless Communication, vol.22, no.5, pp.112-121, Oct. 2015.

[10] B. Di, S. Bayat, L. Song, Y. Li, and Z. Han, "Joint user pairing, subchannel and power allocation in full-duplex multi-user OFDMA networks", IEEE Transaction on Wireless Communication, vol. 15, no. 12, pp.8260-8272, Dec. 2016.

[11] M. Al-Imari, M. Ghoraishi, P. Xiao, and R. Tafazolli, "Game theory based radio resource allocation for full-duplex systems", IEEE VTC Spring, May 2015.

[12] F. Meshkati, H. V Poor, and S. C. Schwartz, "Energy-efficient resource allocation in wireless networks", IEEE Signal Processing Magazine, vol.24, no.3, pp.58-68, May 2007.

[13] C. Zarakovitis, Q. Ni, and J. Spiliotis. "Energy-efficient green wireless communication systems with imperfect CSI and data outage", IEEE Journal on Selected Areas in Communications, vol. 34, issue 12, pp. 3108-3126, Dec. 2016.

[14] M. O. Al-Kadri, A. Aijaz, and A. Nallanathan, "An energy-efficient full-duplex MAC protocol for distributed wireless networks", IEEE Wireless Communications Letters, vol. 5, no. 1, pp.44-47, Feb. 2016.

[15] C. Yang, J. Li, Q. Ni, A. Anpalagan, M. Guizani. "Interference-aware energy efficiency maximization in 5G ultra-dense networks", IEEE Transactions on Communications, vol. 65, issue 2, pp. 728-739, Feb. 2017.

[16] Z. Song, Q. Ni, K. Navaie, S. Hou, S. Wu, and X. Sun, "On the spectral-energy efficiency and rate fairness tradeoff in relay-aided cooperative OFDMA systems", IEEE Transactions on Wireless Communications, vol. 15, issue 9, pp. 6342-6355, Sep. 2016.

[17] H. Xu, W. Xu, Z. Yang, Y. Pan, J. Shi, and M. Chen, "Energy-efficient resource allocation in D2D underlaid cellular uplinks", IEEE Communications Letters, vol.21, no.3, pp.560-563, Mar. 2017.

[18] S. Boyd, and L. Vandenberghe, "Convex optimization", Cambridge, U.K.: Cambridge Univ. Press, 2004.

[19] S. Schaible, "Fractional programming. II, On Dinkelbach's Algorithm", Management Science, vol.22, no.8, pp.868-873, 1976

[20] W. Dinkelbach, "On nonlinear fractional programming", Management Science, vol. 13, no. 7, Mar. 1967.

[21] Z. Han, "Game theory in wireless and communication networks: theory, models, and applications," Cambridge University Press, 2012.

[22] H. W. Kuhn, "The Hungarian method for the assignment", Naval Res. Logist. Quart., vol. 2, no. 1/2, pp. 83-97, Mar. 1955.

[23] T. Kim, and M. Dong, "An iterative Hungarian method to joint relay selection and resource allocation for D2D communications", IEEE Wireless Communications Letter, vol. 3, no. 6, pp. 625-628, Dec. 2014.

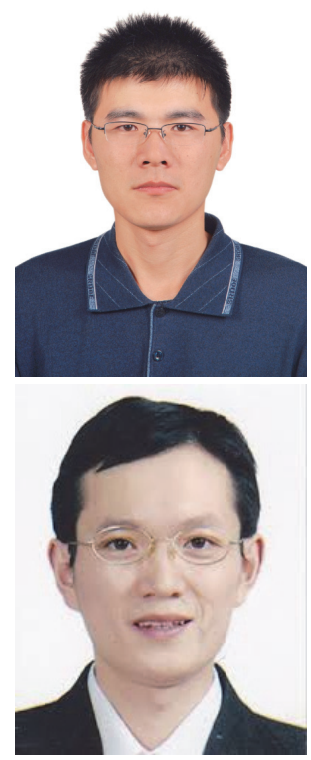

Song Li received the Ph.D. degree in signal and information processing from Beijing University of Posts and Telecommunications, Beijing, China, in 2012. He is currently an Associate Professor with the School of Information and Control Engineering, China University of Mining and Technology, Xuzhou, China. His current research interests include cyber-physical system, full-duplex communications and cooperative communications.

Qiang Ni is a Professor and the Head of Communication Systems Group with InfoLab21, School of Computing and Communications, Lancaster University, Lancaster, U.K. He has authored more than 180 papers in international journals and conferences. His main research interests include the area of future generation communications and networking, including green communications and networking, cognitive radio network systems, heterogeneous networks, 5G, SDN, cyber-physical system, energy harvesting, IoTs and vehicular networks. $\mathrm{He}$ is a Voting Member of IEEE 1932.1 standard. He was an IEEE 802.11 Wireless Standard Working Group Voting member.

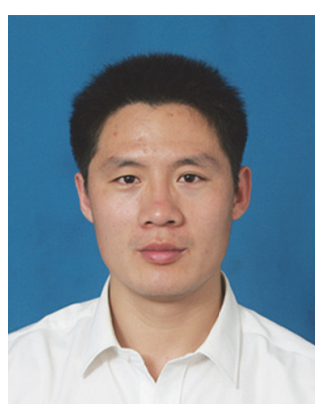

Yanjing Sun received the Ph.D. degree in information and communication engineering from China University of Mining and Technology, Xuzhou, China, in 2008.

$\mathrm{He}$ is currently a Professor and the Vice-Dean of the School of Information and Control Engineering, China University of Mining and Technology. His current research interests include IBFD communications, embedded real-time system, wireless sensor networks and cyber-physical systems.

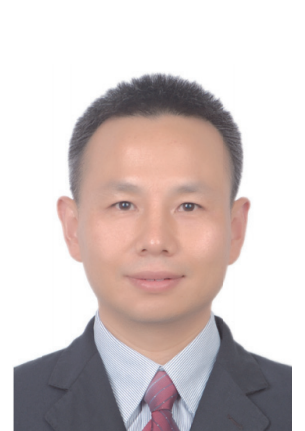

Geyong Min is a Professor of High-Performance Computing and Networking in the Department of Computer Science within the College of Engineering, Mathematics and Physical Sciences at the University of Exeter, United Kingdom. He received the Ph.D. degree in Computing Science from the University of Glasgow, United Kingdom, in 2003, and the B.Sc. degree in Computer Science from Huazhong University of Science and Technology, China, in 1995. His research interests include Future Internet, Computer Networks, Wireless Communications, Multimedia Systems, Information Security, High-Performance Computing, Ubiquitous Computing, Modelling, and Performance Engineering.

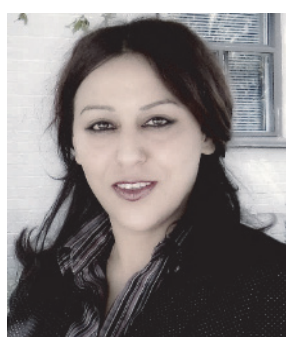

Saba Al-Rubaye received her Ph.D. degree in Electrical and Electronic Engineering from Brunel University London, UK in 2013. She was working with advisory service on several integration, analysis and testing projects at Sustainable Technology Integration Laboratory (QT-STIL), Quanta Technology, Toronto, Canada. She led and managed several projects focusing on Smart Grids, Grid Modernization, SDN Platform, 5G and Beyond Wireless Networks. She is a Chartered Engineer (CEng) registered with Engineering British Council and 